\title{
Tree-Antimagicness of Web Graphs and Their Disjoint Union
}

\author{
Zhijun Zhang, ${ }^{1}$ Muhammad Awais Umar $\mathbb{D}^{2},{ }^{2}$ Xiaojun Ren $\mathbb{D}^{3},{ }^{3}$ Basharat Rehman Ali, ${ }^{4}$ \\ Mujtaba Hussain, ${ }^{5}$ and Xiangmei $\mathbf{L i}^{6}$
}

\author{
${ }^{1}$ School of Computer and Software, Weifang University of Science and Technology, Shouguang 262700, China \\ ${ }^{2}$ Govt. Degree College (B), Sharqpur Sharif, Pakistan \\ ${ }^{3}$ School of Computer and Software, Weifang University of Science and Technology, Shouguang 262700, China \\ ${ }^{4}$ Abdus Salam School of Mathematical Sciences, GC University, Lahore, Pakistan \\ ${ }^{5}$ Department of Mathematics, COMSATS University Islamabad, Lahore, Pakistan \\ ${ }^{6}$ School of Cybersecurity, Chengdu University of Information Technology, Chengdu 610225, China
}

Correspondence should be addressed to Xiaojun Ren; renxiaojun_05@yeah.net

Received 26 January 2020; Accepted 7 March 2020; Published 9 April 2020

Academic Editor: Elio Masciari

Copyright (c) 2020 Zhijun Zhang et al. This is an open access article distributed under the Creative Commons Attribution License, which permits unrestricted use, distribution, and reproduction in any medium, provided the original work is properly cited.

\begin{abstract}
In graph theory, the graph labeling is the assignment of labels (represented by integers) to edges and/or vertices of a graph. For a graph $G=(V, E)$, with vertex set $V$ and edge set $E$, a function from $V$ to a set of labels is called a vertex labeling of a graph, and the graph with such a function defined is called a vertex-labeled graph. Similarly, an edge labeling is a function of $E$ to a set of labels, and in this case, the graph is called an edge-labeled graph. In this research article, we focused on studying super $\left(a_{d}, d\right)-T_{(4,2)}$-antimagic labeling of web graphs $W(2, n)$ and isomorphic copies of their disjoint union.
\end{abstract}

\section{Introduction and Preliminaries}

A $Q$-covering for a finite and simple graph $P$ is a family of subgraphs $Q_{1}, Q_{2}, \ldots, Q_{s}$ with $Q_{t} \cong Q$ and $Q \subset P$, with every edge $E(Q)$ in one of the subgraphs $Q_{t}, t=1,2, \ldots, s$. For an $(l, m)$-graph, a bijection $\alpha: V(P) \cup E(P) \longrightarrow\{1,2, \ldots, l+m\}$ is a total labeling of $P$, and $w t_{\alpha}(Q)=\sum_{v \in V(Q)} \alpha(v)$ $+\sum_{e \in E(Q)} \alpha(e)$ is the corresponding weight of the subgraph Q. Such a graph $P$ is an $\left(a_{d}, d\right)$-Q-antimagic if $w t_{\alpha}(Q)=\left\{a_{d}, a_{d}+d, \ldots, a_{d}+(s-1) d\right\}$, where $a_{d}>0, d \geq 0$ are two integers, $s$ is the number of subgraphs $Q_{t}$ isomorphic to $Q$, and the labeling $\alpha$ is super if vertices are assigned with the integers $\{1,2, \ldots, l\}$.

In [1], Gutiérrez and Lladó defined Q-supermagic labelings and the results: $P_{n}$ and $C_{n}$ which are $P_{h}$-supermagic for some $h$ were proved. Lladó and Moragas [2] investigated $C_{h}$-magicness of wheels $W_{n}$, windmills $W(n, r)$, books $B_{n}$, and of prisms $D_{n}$ for some $h$. In [3], Baca et al. proved results for tree-antimagicness of disconnected graphs. Noshad Ali et al. in [4] discussed the $C_{3}$-antimagicness of corona graphs. Umar [5] stated the construction for antimagicness of ladder graphs. $P_{h}$-magic labelings of trees $T_{n}$, subdivision of shrubs, and banana tree were proved by Maryati, Baskoro, and Salman [6]. The cycle antimagicness of book graphs for differences $\{1,2, \ldots, 13\}$ is given in [7]. Fan graph is cycleantimagic for differences depending upon the length of the cycle which is proved in [8]. Q-antimagicness of the Cartesian product of graphs was discussed by Baca et al. in [9].

Graph labeling has been used in many applications such as coding theory, X-ray crystallography, radar, astronomy, circuit design, communication network addressing, and data base management [10-12]. In the present article, we have studied super $T_{(4,2)}$-antimagic labeling of web graphs $W(2, n)$ for differences $d \in\{0,1, \ldots, 12,14\}$.

\section{Main Results}

The join graph $C_{n}+K_{1}$ is called a wheel graph $W_{n}$, and a helm graph $H_{n}$ is obtained by attaching a pendant edge to 
each cycle vertex of wheel, while the closed helm $\mathrm{CH}_{n}$ is constructed from a helm by joining each pendant vertex to form a cycle. A web graph $W(2, n)$ is the graph obtained from closed helm by attaching a single pendant edge to each vertex of the outer cycle. The vertex and edge set of the web graph are

$$
\begin{aligned}
& V(W(2, n))=\left\{v, a_{(i, j)}: \quad 1 \leq i \leq n, 1 \leq j \leq 3\right\}, \\
& E(W(2, n))=\left\{v a_{(i, 1)}, a_{(i, j)} a_{(i+1, j)}, a_{(i, j)} a_{(i, j+1)}: \quad 1 \leq i \leq n, 1 \leq j \leq 2\right\},
\end{aligned}
$$

where the indices $i$ are taken as modulo $n$.

The vertex $v$ is called the apex or central vertex. The vertices $\left\{a_{(i, 1)}\right\}$ are inner cycle vertices, $\left\{a_{(i, 2)}\right\}$ are outer cycle vertices, and $\left\{a_{(i, 3)}\right\}$ are pendant vertices. The edges $v a_{(i, 1)}$ are spokes, $a_{(i, 1)} a_{(i+1,1)}$ are inner rim edges, $a_{(i, 2)} a_{(i+1,2)}$ are outer rim edges, $a_{(i, 1)} a_{(i, 2)}$ are middle spokes, and $a_{(i, 2)} a_{(i, 3)}$ are outer spokes.

Figure 1 shows a web graph $W(2,6)$.

The web graph is covered by a tree $T_{(4,2)}$ on 6 vertices. The tree $T_{(4,2)}$ is constructed from path $P_{4}$ by joining a pendant edge with two inner vertices. The vertices and edges of $k^{\text {th }}$ tree $T_{(4,2)}^{(k)}$ in a web graph $W(2, n)$ are

$$
\begin{aligned}
V\left(T_{(4,2)}^{(k)}\right)= & \left\{v, a_{(k, 1)}, a_{(k, 2)}, a_{(k, 3)}, a_{(k+1,1)}, a_{(k+1,2)}\right\}, \\
E\left(T_{(4,2)}^{(k)}\right)= & \left\{v a_{(k, 1)}, a_{(k, 1)} a_{(k, 2)}, a_{(k, 2)} a_{(k, 3)},\right. \\
& \left.a_{(k, 1)} a_{(k+1,1)}, a_{(k, 2)} a_{(k+1,2)}\right\} .
\end{aligned}
$$

Figure 2 shows a tree $T_{(4,2)}$.

Each web graph $W(2, n)$ would be covered by $n$ trees of type $T_{(4,2)}$. The weight of $k^{\text {th }}$ tree $T_{(4,2)}^{(k)}$ under a total labeling $\alpha$ would be

$$
\begin{aligned}
w t_{\alpha}\left(T_{(4,2)}^{(k)}\right)= & \sum_{v \in V\left(T_{(4,2)}^{(k)}\right)} \alpha(v)+\sum_{e \in E\left(T_{(4,2)}^{(k)}\right)} \alpha(e) \\
= & \alpha(v)++\alpha\left(v a_{(k, 1)}\right)+\sum_{p=1}^{3} \alpha\left(a_{(k, p)}\right) \\
& +\sum_{p=1}^{2}\left(\alpha\left(a_{(k+1, p)}\right)+\alpha\left(a_{(k, p)} a_{(k, p+1)}\right)\right)+ \\
& +\sum_{p=1}^{2} \alpha\left(a_{(k, p)} a_{(k+1, p)}\right) .
\end{aligned}
$$

Theorem 1. Let $W(2, n)$ define a web graph on $3 n+1$ vertices and $5 n$ edges and $T_{(4,2)}$ be a tree on 6 vertices for $n \geq 3$. Then, $W(2, n)$ admits a super $\left(a_{d}, d\right)-T_{(4,2)}$-antimagic labeling for differences $d \in\{0,2, \ldots, 14\}$.

Proof. The total labeling $\alpha_{d}$ is defined as

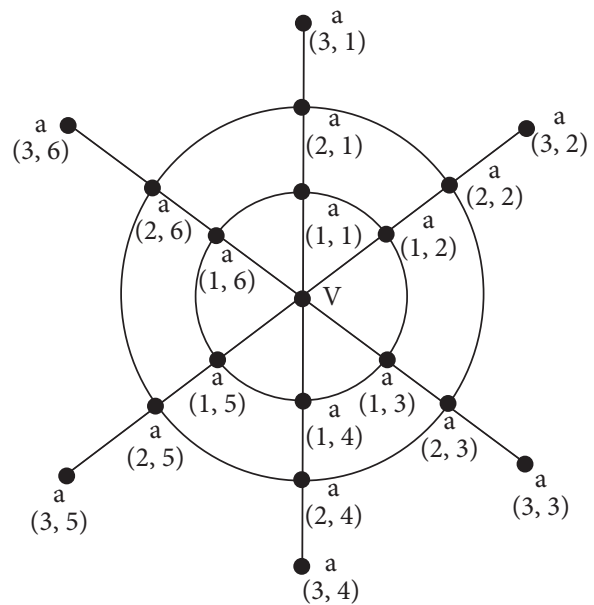

Figure 1: A web graph $W(2,6)$.

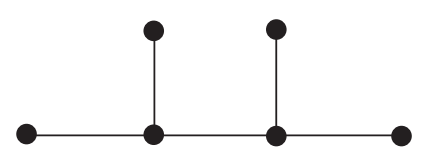

Figure 2: A tree $T_{(4,2)}$.

$$
\begin{aligned}
& \alpha_{d}(\nu)=1 \begin{aligned}
n+2-i, j=1, \\
n+1+i, j=2, \\
2 n+1+i, j=3,
\end{aligned} \\
& \alpha_{d}\left(a_{(i, j)}\right)=\left\{\begin{array}{l}
6 n+2-i, d=0,2, \ldots, 8, \\
3(n+i)+1, d=10,12,14,
\end{array}\right. \\
& \alpha_{d}\left(v a_{(i, 1)}\right)=\left\{\begin{array}{l}
5 n+2-i, j=1, d=0, \\
3 n+1+i, j=2, d=0,2, \\
4 n+1+i, j=1, d=2, \\
3 n+1+2 i, j=1, d=4,6,8, \\
3 n+2 i, j=2, d=4,6,8, \\
3(n+i), j=1, d=10,12,14, \\
3(n+i)-1, j=2, d=10,12,14,
\end{array}\right. \\
& \alpha_{d}\left(a_{(i, j)} a_{(i+1, j)}\right)=\left\{\begin{array}{l}
7 n+2-i, j=1, d=0,2,4, \\
7 n+1+i, j=2, d=0,2,4, \\
6 n+1+i, j=1, d=6,12, \\
7 n+1+i, j=2, d=6,12, \\
6 n+2 i+1, j=2, d=8,14, \\
6 n+1+i, j=1, d=10, \\
8 n+2-i, j=2, d=10,
\end{array}\right.
\end{aligned}
$$

One can easily verify that 


$$
\sum_{j=1}^{3} \alpha_{d}\left(a_{(i, j)}\right)+\sum_{j=1}^{2} \alpha_{d}\left(a_{(i+1, j)}\right)=6 n+7+i .
$$

Using (3) and (5) and the corresponding labeling $\alpha_{d}$, we have

$$
\begin{aligned}
w t_{\alpha_{0}}\left(T_{(4,2)}^{(k)}\right) & =6 n+8+i+(14 n+5-i)+(14 n+3) \\
& =34 n+16 \\
w t_{\alpha_{2}}\left(T_{(4,2)}^{(k)}\right) & =(12 n+10)+(7 n+2+2 i)+(14 n+3) \\
& =33 n+15+2 i \\
w t_{\alpha_{4}}\left(T_{(4,2)}^{(k)}\right) & =(12 n+10)+(6 n+1+4 i)+(14 n+3) \\
& =32 n+14+4 i \\
w t_{\alpha_{6}}\left(T_{(4,2)}^{(k)}\right) & =(12 n+10)+(6 n+1+4 i)+(13 n+2+2 i) \\
& =31 n+13+6 i
\end{aligned}
$$

$$
\begin{aligned}
w t_{\alpha_{8}}\left(T_{(4,2)}^{(k)}\right) & =(12 n+10)+(6 n+1+4 i)+(12 n+1+4 i) \\
& =30 n+12+8 i,
\end{aligned}
$$

$$
\begin{aligned}
w t_{\alpha_{10}}\left(T_{(4,2)}^{(k)}\right) & =6 n+8+i+(9 n+9 i)+(14 n+3) \\
& =29 n+11+10 i
\end{aligned}
$$

$$
\begin{aligned}
w t_{\alpha_{12}}\left(T_{(4,2)}^{(k)}\right) & =6 n+8+i+(9 n+9 i)+(13 n+2+2 i) \\
& =28 n+10+12 i
\end{aligned}
$$

$$
\begin{aligned}
w t_{\alpha_{14}}\left(T_{(4,2)}^{(k)}\right) & =6 n+8+i+(9 n+9 i)+(2 n+1+4 i) \\
& =27 n+9+14 i .
\end{aligned}
$$

Equations (6)-(13) show that $w t_{\alpha}\left(T_{(4,2)}^{(k)}\right)$ constitute an arithmetic progression for differences $d \in\{0,2, \ldots, 14\}$. This completes the proof.

Figures 3 and 4 show the super $(210,4)-T_{(4,2)}$-antimagic labeling of $W(2,6)$ and super $(190,12)-T_{(4,2)}$-antimagic labeling of $W(2,6)$, respectively.

Theorem 2. Let $W(2, n)$ define a web graph on $3 n+1$ vertices and $5 n$ edges and $T_{(4,2)}$ be a tree on 6 vertices for $n \geq 3$. Then, $W(2, n)$ admits a super $\left(a_{d}, d\right)-T_{(4,2)}$-antimagic labeling for differences $d \in\{1,3, \ldots, 11\}$ and for $n \equiv 1(\bmod 2)$.

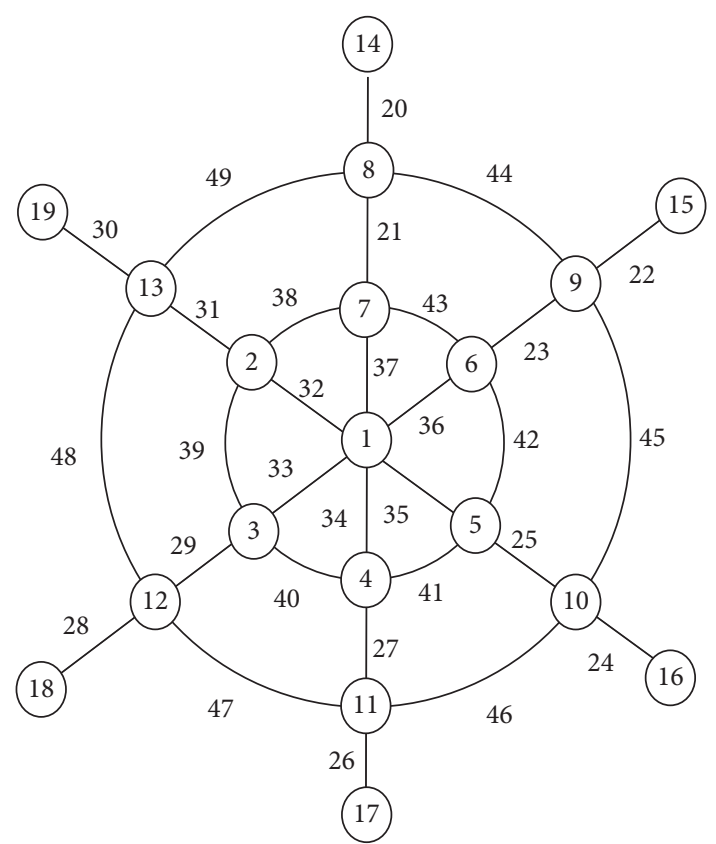

Figure 3: Super $(210,4)-T_{(4,2)}$-antimagic labeling of $W(2,6)$.

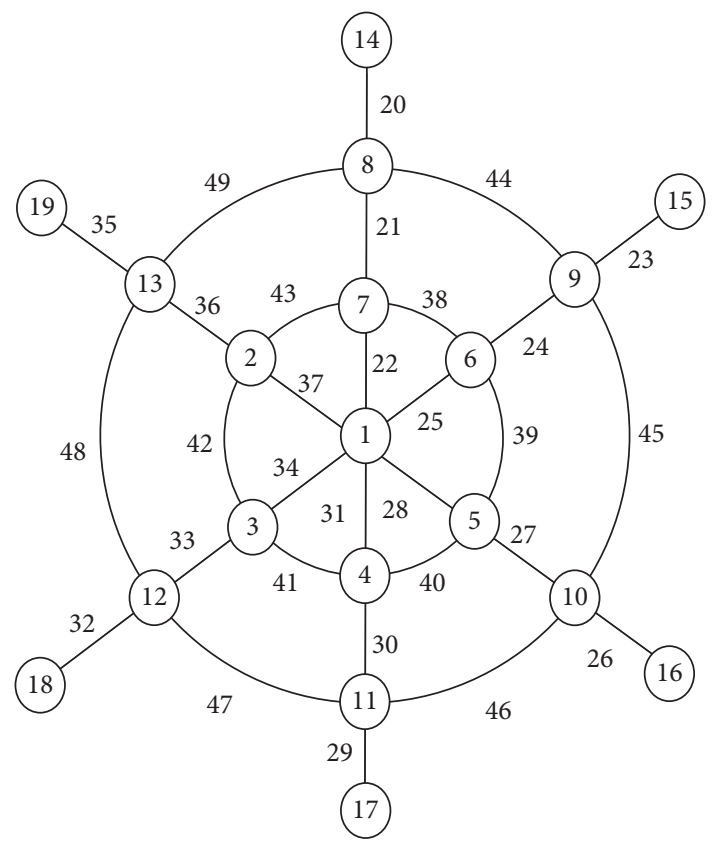

Figure 4: Super $(190,12)-T_{(4,2)}$-antimagic labeling of $W(2,6)$.

Proof. The total labeling $\alpha$ is defined as 


$$
\begin{aligned}
& \alpha_{d}(\nu)=1, \\
& \alpha_{d}\left(a_{(i, j)}\right)=\left\{\begin{array}{l}
1+i, j=1, \quad \text { for all } d, \\
2(n+1)-i, j=2, \quad \text { for all } d, \\
2 n+1+i, j=3, \quad d \neq 9, \\
3 n+2-i, j=3, \quad d=9,
\end{array}\right. \\
& \alpha_{d}\left(v a_{(i, 1)}\right)= \begin{cases}2(2 n+1)-i, & d=1,3, \\
3 n+1+i, & d=5, \\
3 n+2 i, & d=7, \\
3(n+i)-1, & d=9,11,\end{cases} \\
& \alpha_{d}\left(a_{(i, j)} a_{(i, j+1)}\right)= \begin{cases}4 n+1+i, & j=1, d=1,3,5, \\
3 n+1+2 i, & j=1, d=7, \\
3(n+i), & j=1, d=9,11, \\
6 n+2-i, & j=2, d=1, \\
5 n+1+i, & j=2, d=3,5,7, \\
3(n+i)+1 & j=2, d=9,11,\end{cases} \\
& \alpha_{d}\left(a_{(i, 1)} a_{(i+1,1)}\right)= \begin{cases}6 n+1+\frac{i}{2}, & \text { if } i \equiv 0,(\bmod 2), \\
6 n+1+\frac{n-1}{2}+\frac{i+1}{2}, & \text { if } i \equiv 1,(\bmod 2),\end{cases} \\
& \alpha_{d}\left(a_{(i, 2)} a_{(i+1,2)}\right)= \begin{cases}7 n+1+\frac{n+1}{2}+\frac{i}{2}, & \text { if } i \equiv 0,(\bmod 2), \\
7 n+1+\frac{i+1}{2}, & \text { if } i \equiv 1,(\bmod 2) .\end{cases}
\end{aligned}
$$

One can easily verify that

$$
\sum_{j=1}^{3} \alpha_{d}\left(a_{(i, j)}\right)+\sum_{j=1}^{2} \alpha_{d}\left(a_{(i+1, j)}\right)= \begin{cases}4(n+1)+i, & d \neq 9 \\ 5 n+5-i, & d=9 .\end{cases}
$$

Using (3), the above relation, and the corresponding labeling, we have

$$
\begin{aligned}
w t_{\alpha_{1}}\left(T_{(4,2)}^{(k)}\right) & =(20 n+13)+\left(13 n+2+i+\frac{n+1}{2}\right) \\
& =\frac{67 n+31}{2}+i \\
w t_{\alpha_{3}}\left(T_{(4,2)}^{(k)}\right) & =(19 n+12+2 i)+\left(13 n+2+i+\frac{n+1}{2}\right) \\
& =\frac{65 n+29}{2}+3 i \\
w t_{\alpha_{5}}\left(T_{(4,2)}^{(k)}\right) & =(18 n+11+4 i)+\left(13 n+2+i+\frac{n+1}{2}\right) \\
& =\frac{63 n+27}{2}+5 i \\
w t_{\alpha_{11}}\left(T_{(4,2)}^{(k)}\right) & =(15 n+10 i+8)+\left(13 n+2+i+\frac{n+1}{2}\right) \\
& =\frac{57 n+21}{2}+11 i \\
w t_{\alpha_{7}}\left(T_{(4,2)}^{(k)}\right) & =(17 n+10+6 i)+\left(13 n+2+i+\frac{n+1}{2}\right) \\
& =\frac{61 n+25}{2}+7 i \\
w t_{\alpha_{9}}\left(T_{(4,2)}^{(k)}\right)= & (16 n+8 i+9)+\left(13 n+2+i+\frac{n+1}{2}\right) \\
& 59 n+23 \\
&
\end{aligned}
$$

Equations (16)-(21) show that $w t_{\alpha}\left(T_{(4,2)}^{((k)}\right)$ constitute an arithmetic progression for differences $d \in\{1,3, \ldots, 11\}$. This completes the proof.

Figures 5 and 6 show the super $(172,7)-T_{(4,2)}$-antimagic labeling of $W(2,5)$ and super $(168,9)-T_{(4,2)}$-antimagic labeling of $W(2,5)$, respectively.

In view of Theorems 1 and 2 and the construction of the web graph, helm graph, and closed helm graph, one can deduce the following corollaries.

Corollary 1. Let $H_{n}$ define a helm graph on $2 n+1$ vertices and $3 n$ edges and $T_{(3,1)}$ define a tree on 4 vertices for $n \geq 3$. 


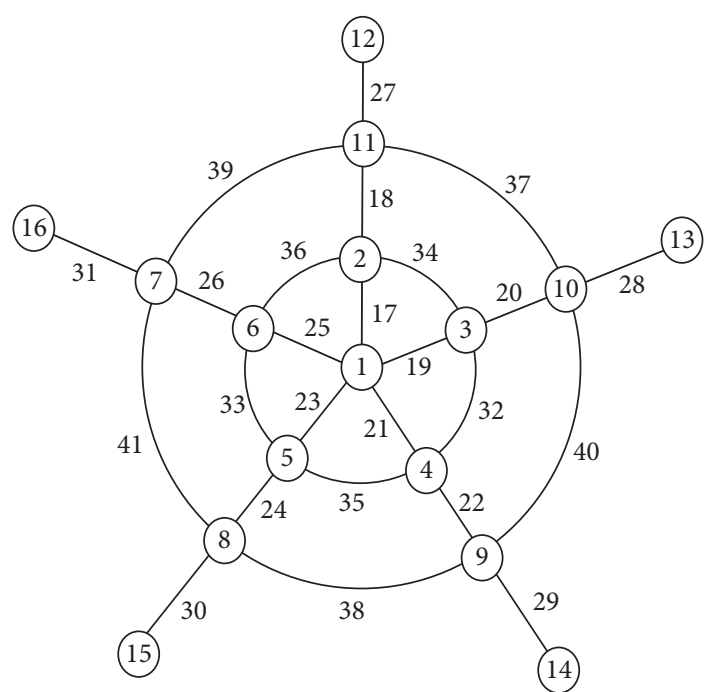

Figure 5: Super $(172,7)-T_{(4,2)}$-antimagic labeling of $W(2,5)$.

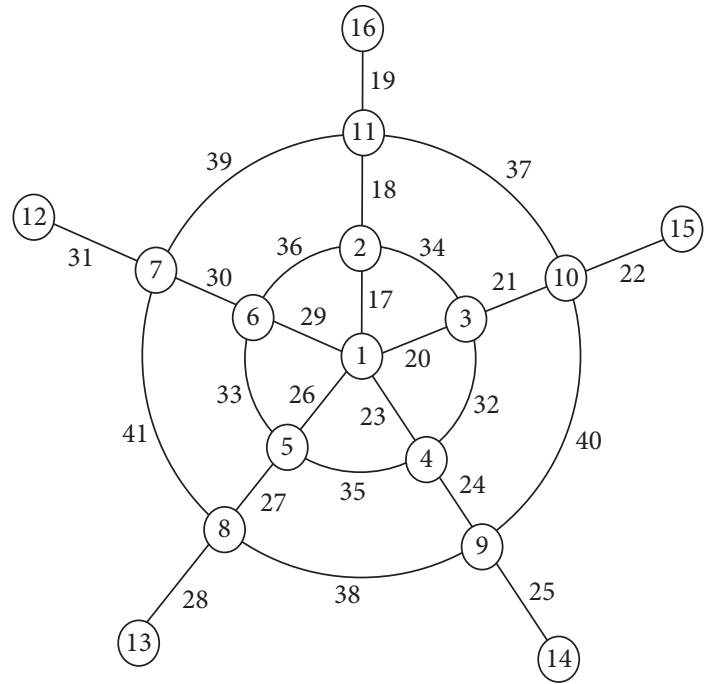

Figure 6: Super $(168,9)-T_{(4,2)}$-antimagic labeling of $W(2,5)$.

Then, $H_{n}$ admits a super $\left(a_{d}, d\right)-T_{(3,1)}$-antimagic labeling for differences $d \in\{0,2, \ldots, 14\}$.

Corollary 2. Let $H_{n}$ define a helm graph on $2 n+1$ vertices and $3 n$ edges and $T_{(3,1)}$ define a tree on 4 vertices for $n \geq 3$. Then, $H_{n}$ admits a super $\left(a_{d}, d\right)-T_{(3,1)}$-antimagic labeling for differences $d \in\{1,3, \ldots, 11\}$ and for $n \equiv 1(\bmod 2)$.

Corollary 3. Let $\mathrm{CH}_{n}$ define a closed helm graph on $2 n+1$ vertices and $3 n$ edges and $T_{(3,2)}$ define a tree on 5 vertices for $n \geq 3$. Then, $\mathrm{CH}_{n}$ admits a super $\left(a_{d}, d\right)-T_{(3,2)}$-antimagic labeling for differences $d \in\{0,2, \ldots, 14\}$.

Corollary 4. Let $\mathrm{CH}_{n}$ define a closed helm graph on $2 n+1$ vertices and $3 n$ edges and $T_{(3,2)}$ define a tree on 5 vertices for $n \geq 3$. Then, $C H_{n}$ admits a super $\left(a_{d}, d\right)-T_{(3,2)}$-antimagic labeling for differences $d \in\{1,3, \ldots, 11\}$ and for $n \equiv 1(\bmod$
2), where tree $T_{(3,2)}$ is obtained from $P_{3}$ by attaching two pendant edges: one pendant edge with the inner vertex and one with the end vertex.

In [3], Bača et al. proved the tree-antimagicness of disconnected graphs. For the complete reference, the results are as follows.

Theorem 3 (see [3]). Let $m, t \geq 1$ be positive integers. Let $G_{i}$ with a $\left(T_{i}^{1}, T_{i}^{2}, \ldots, T_{i}^{t}\right)$-covering be a super (a,1)-T-antimagic graph of order $p$ and size $q$, $i=1,2, \ldots, m$, where $T$ is a tree and every tree $T_{i}^{j}$, $j=1,2, \ldots, t$ is isomorphic to $T$. Then, the disjoint union $\cup_{i=1}^{m} G_{i}$ is also a super $(b, 1)$-T-antimagic graph.

Corollary 5 (see [3]). Let $G$ be a super $(a, 1)$-T-antimagic graph, where $T$ is a tree. Then, the disjoint union of the arbitrary number of copies of $G$, i.e., $m G, m \geq 1$, also admits a super $(b, 1)$-T-antimagic total labeling.

\section{Conclusion}

The first polynomial time construction procedure for different graphs is being discussed in artificial intelligence, for example, see [13]. These procedures and constructions have a wide range of practical applications in radio astronomy, X-ray crystallography, cryptography, and experimental design. In general, many results in theoretical computer science can be generalized and constructed using the graph structures and labeling of the graphs. Here, in this manuscript, we have discussed labeling of web graphs, their associated graphs, and their disjoint union. As a matter of fact, disjoint union of a graph is the relationship between its isomorphic copies keeping the structural properties intact and used to create new larger structures having the same properties as the base graph have. In future, one can extend this idea to find the results for larger differences and for more general structure with applications in theoretical computer science [14-16].

\section{Data Availability}

The data used to support the findings of this study are available from the corresponding author upon request.

\section{Conflicts of Interest}

The authors declare that there are no conflicts of interest.

\section{Acknowledgments}

This work was supported in part by Key Research and Development Program of Shandong under grant 2019GNC106034, Facility Horticultural Laboratory Funding Project in Shandong Universities under grant 2019YY003, and Weifang University of Science and Technology Doctoral Fund Project under grant 2017BS19. 


\section{References}

[1] A. Gutiérrez and A. Lladó, "Magic coverings," Journal of Combinatorial Mathematics and Combinatorial Computer, vol. 55, pp. 43-56, 2005.

[2] A. Lladó and J. Moragas, "Cycle-magic graphs," Discrete Mathematics, vol. 307, pp. 2925-2933, 2007.

[3] M. Bača, Z. Kimáková, A. Semaničová-Feňovčíková, and M. A. Umar, "Tree-antimagicness of disconnected graphs," Mathematical Problems in Engineering, vol. 2015, Article ID 504251, 4 pages, 2015.

[4] N. Ali, M. A. Umar, A. Tabassum, and A. Raheem, "Super (a,d)-C3-antimagicness of a Corona graph," Open Journal of Mathematical Science, vol. 2, no. 1, pp. 371-378, 2018.

[5] M. A. Umar, "Further results on product cordial graphs," International Journal of Mathematics and Soft Computing, vol. 2, no. 2, pp. 67-47, 2019.

[6] T. K. Maryati, A. N. M. Baskoro, and E. T. Salman, ". $P_{h^{-}}$(super)magic labelings of some trees," Discrete Mathematics, vol. 65, no. 4, pp. 198-204, 2008.

[7] G. V. Umar, "Secure domination in lict graphs," Open Journal of Mathematical Sciences, vol. 3, no. 2, pp. 184-190, 2019.

[8] M. A. Ali and N. Ali, "Semaničová-Feňovčíková Fans are cycle-antimagic," Open Journal of Mathematical Sciences, vol. 68, no. 1, pp. 94-105, 2017.

[9] M. Bača and A. Semanicová-fenovcikova, "Des welyyanti and muhammad awais umar, on $H$-antimagicness of cartesian product of graphs," Turkish Journal of Mathematics, vol. 42, pp. 339-348, 2018.

[10] S. Nazeer, I. Kousar, and W. Nazeer, "Radio and radio antipodal labelings for circulant graphs G $(4 k+2 ; 1 ; 2)$," Journal of Applied Mathematics \& Informatics, vol. 33, no. 1-2, pp. 173-183, 2015.

[11] S. M. Kang, S. Nazeer, I. Kousar, W. Nazeer, and Y. C. Kwun, "Multi-level and antipodal labelings for certain classes of circulant graphs," Journal of Nonlinear Sciences and Applications, vol. 9, pp. 2832-2845, 2016.

[12] M. Ajmal, W. Nazeer, M. Munir, S. M. Kang, and Y. C. Kwun, "Some algebraic polynomials and topological indices of generalized prism and toroidal polyhex networks," Symmetry, vol. 9 , no. 1, p. 5, 2017

[13] R. Le Bras, C. P. Gomes, and B. Selman, "Double-wheel graphs are graceful," in Proceedings of the 23rd International Joint Conference on Artificial Intelligence, New York, NY, USA, 2009.

[14] W. Gao, "Remarks on fractional locally harmonious coloring," Open Journal of Mathematical Science, vol. 2018, no. 1, pp. 301-306, 2018.

[15] T. K. Maryati, "Supermagic coverings of the disjoint union of graphs and amalgamations," Open Journal of Mathematical Sciences, vol. 2018, pp. 397-405, 2013.

[16] H. M. Nagesh and M. C. Mahesh Kumar, "Block digraph of a directed graph," Open Journal of Mathematical Science, vol. 2, no. 1, pp. 202-208, 2018. 